\title{
A Survey on Age Estimation Techniques
}

\author{
Somy Soman \\ M.Tech Student, Viswajyothi College Of \\ Engineering and Technology
}

\author{
Amel Austine \\ Assistant Professor, Viswajyothi College Of \\ Engineering and Technology
}

\begin{abstract}
Age is an important trait used for identity authentication. The factors that affect aging process include a person's gene, health, living style etc. Age Estimation is predicting a person's age. Out of these, face is the most convenient one. Age Estimation has lots of real-world applications, such as security control, biometrics, customer relationship management, entertainment and cosmetology. In this paper, we compare some of the techniques used in the age estimation based on face images. The most commonly used database is FG-NET. The most commonly used age estimation method is regression based because it takes into account the interrelationship among the age values. Age Estimation via Grouping and Decision Fusion provides minimum MAE, 2.81 for FG-NET and 2.97 for MORPH II.
\end{abstract}

\section{Keywords}

AGES, MAE, ANOVA, GOP

\section{INTRODUCTION}

Human face provides a lot of useful information about a person such as age, gender, expression, etc. Facial age estimation has gained wide popularity in recent years. It has a lot of applications in the daily life of a person. They include electronic vending machines, forensic art, entertainment, cosmetology, etc. Even if the field of age estimation has grown a lot, it is still challenging to estimate age because the process of aging is affected by factors like change in shape and size of face, living style, eating habits, environment, etc. Furthermore, the presence of surgical marks, spectacles, cap, facial scars, facial cosmetics and beard can negatively affect the accuracy of facial age estimation systems. Non frontal head pose also affect the accuracy of age estimation systems.

Age estimation from human faces is a challenging problem. It has applications in forensics, security control, biometrics, electronic customer relationship management, entertainment, cosmetology, etc. The frequently used measure of age estimation is the mean absolute error (MAE). It is the average of absolute error between estimated age and ground truth age. The main challenge of age estimation is that the facial feature changes due to aging are different for different humans.

Gender plays an important role in the aging process because aging patterns and features are different in males and females. Age estimation is a topic that is active today because this information needs to be included in real-world systems. Age is important to understand requirements of a person in different aspects of his daily life. Some systems implement age specific human computer interaction. They are vending machines, advertisements etc. Automatic facial age estimation is affected by traditional factors. Unknown illumination conditions, non-frontal facial poses, and presence of facial expressions, are some factors. Especially, facial expressions like smile might negatively affect accuracy of automated systems: When a person smiles wrinkles are formed. These wrinkles can be misleading when only the appearance features are taken into account.

\section{LITERATURE SURVEY}

Xin, Zhi, and Kate [1] proposed an approach called AGES (AGing pattErn Subspace). AGES considers each aging pattern as a sample. The aging patterns are modelled by a representative subspace. A point in the subspace corresponds to an aging pattern. The proper aging pattern for a face image that is previously unseen is determined by the projection of that image in the subspace. Once the aging pattern is determined, the position of the face in the aging pattern will then indicate its age.

Yun and Thomas [2] proposed an age estimation framework which consists of face detection, discriminative manifold learning, and multiple linear regressions. An automatic face detector is used to detect and crop the face image patches. Some standard alignments are then performed to normalize the features. The discriminative aging manifold is learned. It is done to map the original data into a low-dimensional subspace that is sufficiently discriminative. A multiple linear regression procedure is used with a quadratic function. It is used to estimate a statistical age model over the lowdimensional representation.

Guodong, Yun, and Thomas [3] proposed an age estimation framework which consists of face detection, face normalization, manifold learning, robust regression, and local adjustment. The face image patches are automatically detected and cropped from images by face detection. A face detector is used to find the face area in each image, and label the eye corner locations of each face subject. The face images then undergo cropping, scaling, and they are transformed to $60 *$ 60 gray-level patches. The grey-level values of each face image are normalized. They are normalized to a normal distribution. Normalization is performed on cropped face patches. Normalization includes geometric alignments and illumination normalization. Then the age manifold is learned to map the original face image data into a low-dimensional subspace. After learning the age manifold, each face image is projected onto the age manifold. Then a feature vector is extracted. A robust regression function is applied to fit the manifold data.

Jinli, Song, Shiguang, and Xilin [4] proposed a compositional and dynamic model to represent the face aging process. The model represents faces in each age group by a three-level And-Or graph. It consists of And nodes, Or nodes, and Leaf nodes. The And nodes represent the decomposition. It divides a face into parts and primitives at three levels from coarse to fine. The first level describes appearance of face and hair. At the second level the facial components are refined. The wrinkles and skin marks are refined at the third level. Or nodes represent the diversity of face appearance at each age group. Leaf nodes are basic primitives. By selecting alternatives at the Or nodes, one obtains a hierarchic parse graph for a face instance. The face image can be synthesized from this parse graph. The dynamics of the face aging process is represented as a first-order Markov chain on parse graphs 
and learn the aging patterns from annotated faces of adjacent age groups at each level. The compositional model decomposes face into facial components and skin zones.

Kuan, Shuicheng, and Jay [5] proposed the age estimation scheme in which the age grouping method is used to classify face images into different age groups. The entire age range is divided into several non-overlapping ranges and each age group has a different range. Then, the gradient orientation pyramid (GOP) is adopted to represent overall facial features. To further increase the discriminating ability of the feature space, the analysis of variance (ANOVA) is employed to select the more discriminative features from the GOP feature vector, which significantly reduces the dimensionality of the GOP feature vector. Then, the linear support vector machine (SVM) is adopted to learn a model and classify faces into age groups. The exact age for each face is estimated within its group range. Both local and global features are used. Local features are obtained by extracting features from local facial areas. A cascaded object detector using the Viola-Jones algorithm is adopted to detect three facial components (eyes, nose and mouth). The global features are obtained by extracting BIF, HOG, and LBP from the whole face. Every global or local feature is used by the support vector regression (SVR) to predict ages for faces in each group. Finally, decisions from the system outputs are fused.

Hamdi, Fares, Albert, and Theo [6] proposed an age estimation scheme in which a mesh model is fitted to face using 17 fiducial points, and tracked during the rest of the video. The surface deformations are computed using the tracked mesh points. Temporal phases (onset, apex, and offset) of the expression are estimated. For this the mean displacement signal of the lip corners is used. Dynamic features are extracted from each regional patch from each phase. Appearance features(IEF,GEF[8],BIF[9],LBP[10]) are extracted from first frame of the onset phase. The most informative dynamic features are selected using a feature selection procedure and fused with appearance features to train Support Vector Machine (SVM) classifiers/regressors.

Table 1 summarizes and compares some existing age estimation techniques.

Table 1: Comparison of Age Estimation Techniques

\begin{tabular}{|c|c|c|c|c|c|}
\hline S. No & Paper Name & Database & Feature Extraction & Algorithm & Performance(MAE) \\
\hline 1 & $\begin{array}{c}\text { Facial Aging } \\
\text { Patterns [1] }\end{array}$ & $\begin{array}{c}\text { FG-NET and } \\
\text { MORPH }\end{array}$ & $\begin{array}{c}\text { Holistic appearance, } \\
\text { principal component } \\
\text { analysis[7] (PCA) }\end{array}$ & Regression & $\begin{array}{l}\text { FG-NET:6.8 } \\
\text { MORPH: } 8.8\end{array}$ \\
\hline 2 & $\begin{array}{c}\text { Regression on } \\
\text { Discriminative } \\
\text { Aging Manifold } \\
{[2]}\end{array}$ & YGA & CEA & Regression & $\begin{array}{c}\text { Female:5.5 } \\
\text { Male: } 6.0\end{array}$ \\
\hline 3 & $\begin{array}{c}\text { Manifold } \\
\text { Learning and } \\
\text { Locally } \\
\text { Adjusted } \\
\text { Robust } \\
\text { Regression [3] }\end{array}$ & FG-NET & OLPP & Regression & $\begin{array}{c}\text { Female:7.8 } \\
\text { Male: } 8.0\end{array}$ \\
\hline 4 & $\begin{array}{l}\text { Compositional } \\
\text { and Dynamic } \\
\text { Model [4] }\end{array}$ & FG-NET & $\begin{array}{l}\text { Holistic and local } \\
\text { topology, 2D shape, } \\
\text { color, and gradient }\end{array}$ & Regression & 6.0 \\
\hline 5 & $\begin{array}{c}\text { Grouping and } \\
\text { Decision Fusion } \\
{[5]}\end{array}$ & $\begin{array}{c}\text { FG-NET and } \\
\text { MORPH II }\end{array}$ & BIF,HOG and LBP & $\begin{array}{l}\text { Classification } \\
\text { and Regression }\end{array}$ & $\begin{array}{c}\text { FG-NET:2.81 } \\
\text { MORPH II:2.97 }\end{array}$ \\
\hline 6 & $\begin{array}{c}\text { Combining } \\
\text { Facial } \\
\text { Dynamics With } \\
\text { Appearance [6] }\end{array}$ & $\begin{array}{c}\text { UVA-NEMO } \\
\text { Smile } \\
\text { Database and } \\
\text { UVA-NEMO } \\
\text { Disgust } \\
\text { Database }\end{array}$ & $\begin{array}{c}\text { Dynamic Features and } \\
\text { Appearance } \\
\text { Features(IEF,GEF,BIF } \\
\text { and LBP) }\end{array}$ & $\begin{array}{l}\text { Classification } \\
\text { and regression }\end{array}$ & $4.33-4.77$ \\
\hline
\end{tabular}

\section{FINDINGS}

Many databases are available for age estimation. The most commonly used database is FG-NET The reason for its popularity is that, it is a baseline database for comparisons with many existing age estimation techniques. The most commonly used age estimation method is regression based. The classification based methods overlook the interrelationship among the age values. The regression approaches take into account the inter-relationship among the age values. 
But a more promising approach is to combine regression and classification methods so that the accuracy might be improved further. Age Estimation via Grouping and Decision Fusion provide the minimum MAE, 2.81 for FG-NET and 2.97 for MORPH II.

\section{CONCLUSION}

Age Estimation is a broad area wherein many studies and researches have been carried out. There are many problems in existing systems that need to be addressed such as occlusion, uneven illumination. However some of the problems like non frontal pose, collection of images along with their age on large scale have already been solved. The age estimation method can be either classification based or regression-based. For databases with sequential age labels, both classification based and regression based can be applied, while for databases with only age group labels, classification-based methods can be applied. However, it is also reasonable to consider a hybrid of these two.

\section{REFERENCES}

[1] Xin Geng, Zhi-Hua Zhou, Kate Smith-Miles, "Automatic Age Estimation Based on Facial Aging Patterns," IEEE Trans. On Pattern Analysis And Machine Intelligence, vol. 29, no.12, December 2007.

[2] Yun Fu and Thomas S. Huang, "Human Age Estimation With Regression on Discriminative Aging Manifold," IEEE Trans. On Multimedia, vol. 10, no. 4, June 2008.

[3] Guodong Guo,Yun Fu Charles R. Dyer, and Thomas S. Huang, "Image-Based Human Age Estimation by Manifold Learning and Locally Adjusted Robust
Regression," IEEE Trans. On Image Processing, vol. 17, no. 7, July 2008.

[4] Jinli Suo, Song-Chun Zhu, Shiguang Shan, and Xilin Chen, "A Compositional and Dynamic Model for Face Aging," IEEE Trans. On Pattern Analysis And Machine Intelligence, vol. 32, no. 3, March 2010.

[5] Kuan-Hsien Liu, Shuicheng Yan, and C.-C. Jay Kuo, "Age Estimation via Grouping and Decision Fusion," IEEE Trans. On Information Forensics and Security, vol. 10, no. 11, November 2015.

[6] Hamdi Dibeklioglu, Fares Alnajar, Albert Ali Salah, and Theo Gevers, Zhou, "Combining Facial Dynamics With Appearance for Age Estimation," IEEE Trans. On Image Processing, vol. 24, no. 6, June 2015.

[7] I.T. Jolliffe, Principal Component Analysis, second ed. Springer-Verlag, 2002.

[8] F. Alnajar, C. Shan, T. Gevers, and J.-M. Geusebroek, "Learning-based encoding with soft assignment for age estimation under unconstrained imaging conditions," Image Vis. Comput., vol. 30, no. 12, pp. 946-953,2012.

[9] G. Guo, G. Mu, Y. Fu, and T. S. Huang, "Human age estimation using bio-inspired features," in Proc. IEEE CVPR, Jun. 2009, pp. 112-119.

[10] T. Ojala, M. Pietikäinen, and D. Harwood, “A comparative study of texture measures with classification based on featured distributions," Pattern Recognit., vol. 29 , no. 1 , pp. 51-59, 1996. 